\title{
Recomendaciones latinoamericanas de consulta segura en dermatología
}

\section{Latin American recommendations for safe consultation in dermatology}

\author{
Jorge Ocampo-Candiani ${ }^{*}, M^{a}$ Ivonne Arellano-Mendoza $^{2}$, Roberto Arenas ${ }^{3}$, Angélica Beirana-Palencia ${ }^{4}$, \\ Horacio A. Cabo ${ }^{5}$, Emilia N. Cohen-Sabban ${ }^{6}$, Manuel del Solar, Judith Domínguez-Cherit ${ }^{7}$, Félix Fich ${ }^{9}$, \\ Gastón Galimberti10, Carlos Fernando Gatti11, Carlos García-Rementería ${ }^{12}$, José L. López-Estebaranz ${ }^{13}$, \\ Omar Lupi ${ }^{14}$, Miguel Olmos-Pérez ${ }^{15}$, Ricardo Pérez-Alfonzo ${ }^{16}$, César J. Ramos-Cavazos ${ }^{17}$ y \\ Alfredo Salmon-Demongin ${ }^{18}$
}

${ }^{1}$ Departamento de Dermatología, Facultad de Medicina y Hospital Universitario, Universidad Autónoma de Nuevo León, Monterrey, México; 'Departamento de Dermatología, Hospital General de México, Ciudad de México, México; 'Sección de Micología, Hospital "Manuel Gea González", Secretaría de Salud, Ciudad de México, México; ${ }^{4}$ Sociedad Mexicana de Dermatología, Ciudad de México, México; ${ }^{5}$ Departamento de Dermatología, Universidad de Buenos Aires, Buenos Aires, Argentina; ${ }^{6}$ Servicio de Dermatología, Instituto de Investigaciones Médicas Alfredo Lanari, Universidad de Buenos Aires, Buenos Aires, Argentina; ${ }^{7}$ Servicio de Dermatología, Hospital Cayetano Heredia, Lima, Perú; ${ }^{8}$ Departamento de Dermatología, Instituto Nacional de Ciencias Médicas y Nutrición "Salvador Zubirán", Ciudad de México, México; ${ }^{9}$ Departamento de Dermatología, Pontificia Universidad Católica de Chile, Santiago, Chile; ${ }^{10}$ Sección de Oncología Cutánea y Cirugía de Mohs, Hospital Italiano, Buenos Aires, Argentina; ${ }^{11}$ Instituto de Medicina Cutánea de Buenos Aires, Buenos Aires, Argentina; ${ }^{12}$ Dermatología Clínica y Quirúrgica, Southwestern Dermatology, Oklahoma City, Oklahoma, EE.UU.; ${ }^{13}$ Servicio de Dermatología, Hospital Universitario Fundación Alcorcón, Madrid, España; ${ }^{14}$ Policlínica Geral do Rio de Janeiro (PGRJ), Universidade Federal do Rio de Janeiro (UFRJ), Rio de Janeiro, Brazil; ${ }^{15}$ Departamento de Dermatología, Fundación Universitaria de Ciencias de la Salud (FUCS), Bogotá, Colombia; ${ }^{16}$ Departamento de Dermatología, Instituto de Biomedicina, Universidad Central de Venezuela, Hospital Vargas Clínica El Ávila, Caracas, Venezuela; ${ }^{17}$ Departamento de Dermatología, Facultad de Medicina y Hospital Universitario, Universidad Autónoma de Nuevo León, Monterrey, México; ${ }^{18}$ Departamento Editorial, Latin American Journal of Clinical Sciences and Medical Technology, Ciudad de México, México

\section{Resumen}

Objetivo: Fortalecer la protección a la salud tanto de los pacientes como del especialista y de su personal de apoyo, ya que la pandemia de COVID-19 impone modificaciones a la práctica médica. Material y métodos: Un grupo de expertos dermatólogos latinoamericanos emite recomendaciones para la práctica dermatológica segura, dentro del escenario de la pandemia de COVID-19, en lo que respecta a información básica, capacitación y supervisión en protección personal, antecedentes del paciente, manejo de expedientes, mejoramiento en las áreas no médicas relacionadas con el control del consultorio, junto con la vigilancia de probables casos de contagio en los pacientes. Resultados: Las recomendaciones abarcan las actividades antes, durante y después de la consulta. Se tratan aspectos como el arreglo del consultorio, el control del flujo de trabajo, los procedimientos de asepsia, el tiempo y la atención de las áreas dermatológicas o el uso de los medios digitales para la atención. Conclusiones: El diseño de protocolos adecuados depende de que los especialistas comprendan las características del comportamiento de casos de COVID-19 en su localidad y mantengan contacto con autoridades de salud y centros de atención a síntomas para determinar los tiempos de cierre y reapertura del consultorio.

Palabras clave: COVID-19. SARS-Cov-2. Dermatología. Prevención. Telemedicina.

Correspondencia:

*Jorge Ocampo-Candiani

E-mail: jocampo2000@yahoo.com.mx
Disponible en internet: 14-12-2020 Med Cutan Iber Lat Am. 2020;48(2):88-94 www.MedicinaCutanealLA.com 0210-5187/@ 2020 Colegio Ibero Latinoamericano de Dermatología A.C. (CILAD). Publicado por Permanyer. Este es un artículo open access bajo la licencia CC BY-NC-ND (http://creativecommons.org/licenses/by-nc-nd/4.0/). 


\section{Abstract}

Objective: Strengthening health protection for patients, as well as for the specialist and their support staff because the COVID-19 pandemic imposes modifications to medical practice. Material and methods: A group of Latin American dermatology experts designated by the Mexican Society of Dermatology and the Ibero-Latin American College of Dermatology (CILAD) issued recommendations for safe dermatological practice within the scenario of the COVID-19 pandemic, regarding basic information, training, and supervision in personal protection, patient history, file management, improvement in non-medical areas related to the control of the consultation room, along with the surveillance of probable cases of infection in patients. Results: The recommendations cover the activities before, during, and after the consultation. Aspects such as office arrangement, work flow control, asepsis, time and care of dermatological areas and the use of digital media are discussed. Conclusions: The design of adequate protocols depends on the specialists understanding the characteristics of the behavior of COVID-19 cases in their location, maintaining contact with health authorities and symptom attention centers, to determine the closing and reopening times of the clinic.

Key words: COVID-19. SARS-Cov-2. Dermatology. Prevention. Telemedicine.

\section{Introducción}

La dermatología, sea en su práctica privada o en la institucional, ha suspendido la atención de muchos pacientes durante la pandemia de COVID-19. Diversas organizaciones en el mundo han emitido recomendaciones para una práctica segura de la medicina y la dermatología en estos tiempos, pero es deseable y necesario contar con recomendaciones adaptadas al medio latinoamericano y dar a éstas la difusión suficiente para asegurar su implementación ${ }^{1}$. Para ello, la Sociedad Mexicana de Dermatología y el Colegio IberoLatinoamericano de Dermatología (CILAD) designaron a un comité de expertos latinoamericanos para redactar un texto preliminar que adaptara las recomendaciones internacionales a la región y, a continuación, consensuara el documento hasta su formato final.

Estas recomendaciones para la reapertura de consultorios abarcan tres etapas ${ }^{1}$.

I. Antes de la consulta:

1. Información básica para el personal de la clínica sobre las regulaciones y requerimientos locales.

2. Capacitación y supervisión de todo el personal en cuanto a la higiene de manos y el uso correcto de EPP (equipo de protección personal).

3. Elaboración de un cuestionario para pacientes antes de su cita (antecedentes de infección por COVID-19 o de contactos positivos).

4. Instrumentación del expediente clínico presencial o a distancia (teledermatología o entrevista virtual).

5. Habilitación de las áreas administrativas y área de recepción de pacientes, incluidos procedimientos y técnicas de desinfección.

II. Durante la consulta

1. Protocolo para admitir/recibir al paciente en la clínica (algunas clínicas toman la temperatura de todos los pacientes y no los admiten si tienen fiebre o tosen; la mayoría exige uso de mascarilla para todos los pacientes).

2.Preparación de las áreas de recepción y sala de espera (distanciamiento social, remoción de todos los materiales de lectura o juguetes, gel desinfectante en abundancia).

3. Protocolos para procedimientos quirúrgicos o invasivos.

III. Después de la consulta

1. Adoptar un procedimiento de desinfección de los consultorios después de la consulta.

2. No interrumpir las consultas a distancia.

3. Reducir al mínimo el seguimiento en persona.

4. Considerar el seguimiento a distancia cuando sea práctico.

Estas recomendaciones representan una guía general que deben actualizar de forma periódica los médicos especialistas, de acuerdo con su grado de riesgo profesional y personal de padecer COVID-19 y en concordancia con las regulaciones de su país y localidad.

\section{Recomendaciones ${ }^{1}$}

I. Antes de la consulta

1. Información básica. La enfermedad producida por el Sars-CoV-2 es una afección nueva. El conocimiento de este virus y su comportamiento en el ser humano, afortunadamente, evoluciona día con día. Es vital conocer la tasa de prevalencia del COVID-19 en la comunidad. Las comunidades con mayor prevalencia requieren que las prácticas de apertura a la actividad esencial de la consulta dermatológica se realicen a más largo plazo, mientras que las de una incidencia menor de COVID-19 podrían reanudar antes. 
2. Consultar con la autoridad local y estatal para conocer las disposiciones sanitarias locales.

3. Mantenerse atento a las actualizaciones de las autoridades de salud pública en cuanto a casos de transmisión de COVID-19. Si surge un aumento de casos, instituir una conducta establecida para determinar si las instalaciones deberán reducir los servicios de forma temporal.

4. Contar con un plan para pacientes que acudan con síntomas de COVID-19 y necesiten pruebas diagnósticas de la enfermedad, de laboratorio o de imagen para valorar la gravedad de la enfermedad. Conocer la localización de los lugares donde se realicen las pruebas diagnósticas cercanos al área para enviar a los pacientes a realizarlas o referirlos a su médico de atención primaria. De acuerdo con la definición del Centro de Control de la Enfermedad (CDC) del riesgo de exposición a Sars- CoV 2 , las prácticas en dermatología se incluirán en la categoría de bajo riesgo. Es importante tener un cuestionario sobre síntomas de COVID-19 y enviarlo a cada paciente antes de la consulta para identificar posibles casos sintomáticos y solicitar pruebas diagnósticas.

5. Limpiar y desinfectar el local de acuerdo con los estándares de la Organización Mundial de la Salud: alcohol etílico al 70\% para desinfectar las áreas pequeñas entre usos, así como el equipo reutilizable (p. ej., termómetros); o hipoclorito de sodio al $0.5 \%$ (equivalente a $5,000 \mathrm{ppm}$ ) para desinfectar superficies.

6. Si la consulta ha estado cerrada por más de siete días es posible hacer la limpieza regular, ya que el CDC señala que el virus que causa el COVID-19 no ha demostrado sobrevivir en superficies por más de ese tiempo.

7. Reorganizar la consulta para reducir al mínimo el contacto con el paciente; colocar letreros en sitios visibles para notificar a los pacientes de las precauciones contra COVID-19 y añadir marcas donde sea necesario para mantener la distancia social apropiada $(1.8 \mathrm{~m})$ (como señalamientos con cinta frente a la recepción para que los pacientes mantengan la distancia entre ellos y con el personal). Reducir la cantidad de sillas de la sala de espera y separarlas apropiadamente $(1.8 \mathrm{~m}$.). Retirar las revistas y material de lectura de las áreas de pacientes. Colocar desinfectantes para manos y toallitas adicionales en la sala de espera y las áreas de mucho tránsito para el personal. Disponer de desinfectante para manos o instalaciones para lavárselas con agua y jabón en todos los cuartos. Considerar mantener todas las puertas abiertas en el camino del paciente desde su entrada hasta su salida. Revisar los estándares de EPP y asegurarse de contar con suficiente EPP para todo el personal. Instalar si es posible mamparas transparentes entre su personal secretarial y entre éste y los pacientes puesto que al acercarse a pagar la consulta se reduce considerablemente la distancia.

8. Notificar a los pacientes las medidas que el consultorio ha tomado para mantenerlos seguros. Flexibilizar la cancelación porque los pacientes pueden temer una visita durante esta temporada.

9. Organizar al personal. Según el tamaño de la práctica, el número de personal puede variar en considerable medida. Deben seguirse las actualizaciones y revisar con las autoridades de salud de su estado y localidad las regulaciones de reuniones de grupo. Tratar de limitar la cantidad de personal por cuarto.

10. Educar al personal en cuanto a la distancia social en cuartos de descanso o áreas de comer para que se mantengan al menos a $1.80 \mathrm{~m}$ de distancia. Proporcionar entrenamiento suficiente sobre el uso del EPP, las medidas y protocolos adoptados por su consulta e instituir una forma de supervisar su adecuada realización.

11. El personal debe portar EPP para las reuniones 0 sentarse por lo menos a $1.5 \mathrm{~m}$ de distancia entre cada uno.

12. Informar que no deben acudir a la práctica si presentan síntomas de tipo gripal o pérdida del gusto $\mathrm{u}$ olfato y deben seguir el criterio de sus autoridades sanitarias para el regreso al trabajo.

13. El personal con síntomas indicativos de infección por COVID-19, o los que hayan estado en contacto con ellos, no deben acudir a trabajar y deben confinarse y seguir los lineamientos locales. Sin embargo, es importante recordar que si portan EPP y mantienen distanciamiento social durante toda la jornada, no deben considerarse como un foco infeccioso para las demás personas (personal de trabajo o pacientes).

14. Notificar a sus pacientes cuándo se reabrirá la consulta y sus horarios. Informar la fecha de reapertura para procedimientos electivos a través de las redes sociales, página web y otros canales de mercadotecnia. Dar a conocer los pasos que se han tomado para prevenir las infecciones por COVD-19 en la consulta. Tratar de reanudar con el menor número de pacientes de lo normal para 
ajustarse a los pasos adicionales tomados para la seguridad del personal y los pacientes hasta que se vuelva un esquema sistemático. Hay que estar preparado para tomar cualquier paso necesario si resurgen los casos en la comunidad o clínica luego de la reapertura.

II. Durante la consulta

1. Considerar instituir el pago mediante transferencia bancaria, compartir con los pacientes por vía electrónica los datos de su cuenta para depósito o utilizar las aplicaciones para teléfono móvil correspondientes de cada banco. Otra opción consiste en usar tarjetas Sin Contacto (Contactless), de tal forma que pueda limitarse la manipulación de efectivo y proteger al personal.

2. Vigilar a los pacientes respecto de síntomas de tipo gripal, pérdida del gusto $u$ olfato e historial de exposición al COVID-19; considerar también la toma de temperatura sin contacto. Si hay sospecha de COVID-19, referir al paciente a su médico de atención primaria para su valoración y reprogramar su cita para otra fecha.

3. Hacer esperar a los pacientes afuera de la oficina hasta que corresponda su turno avisando por medio del teléfono móvil. Los pacientes tendrán la consulta solos, a menos que requieran de un cuidador (o padre en caso de niños) con ellos. Otros acompañantes deben esperar en otro sitio o afuera de la oficina.

4. El personal que trata con los pacientes debe portar mascarilla y protección para los ojos y los pacientes deben usar una mascarilla de tela o desechable.

5. Emplear herramientas digitales para favorecer la práctica y maximizar la distancia social cuando sea apropiado: si tiene un registro electrónico de salud (EHR), llamar al proveedor para confirmar si hay aplicaciones instalables para reducir el contacto en persona. Por ejemplo, hay portales de pacientes, pago de facturas en línea, órdenes electrónicas para el personal, recetas y órdenes de laboratorio electrónicas. Debe continuarse la teledermatología para los pacientes en quienes sea apropiado.

6. Organizar el horario, incluidas las visitas de telemedicina. El dermatólogo tratante decidirá qué visitas pueden realizar a distancia y cuáles requieren ser presenciales.

7. Programar con prioridad los casos que exigen la mayor urgencia durante el tiempo en que la consulta estuvo cerrada o limitada a servicios esenciales y que no pudieron atenderse en persona.
8. Antes de ver a pacientes, preguntar cada día a su personal por síntomas de tipo gripal (tos, fiebre, dolor faríngeo o dificultad respiratoria), y pérdida de gusto $u$ olfato o contacto con posibles pacientes con COVID-19. Considerar la tomas de temperatura sin contacto. Si los hallazgos sugieren la posibilidad de infección por COVID-19, referir al personal a su médico de atención primaria para su valoración.

9. Ser flexible con el personal cuando sea posible. Las opciones de guardería y escuela pueden estar limitadas durante este tiempo. Estos nuevos procedimientos deben comunicarse al personal antes de reabrir o aumentar la práctica. Comprender las consideraciones legales de empleo durante la pandemia.

10. Determinar el flujo de trabajo preconsulta junto con el cuestionario de COVID-19 y los pasos para reducir al mínimo el contacto con el paciente. Antes de llegar a la cita o el día anterior, confirmar con el paciente si ha tenido cualquier síntoma de infección respiratoria (tos, fiebre o dificultad para respirar) o pérdida del gusto $u$ olfato. De manera adicional, preguntarle al paciente si ha tenido contacto cercano con pacientes diagnosticados o expuestos a $\mathrm{CO}$ VID-19. Si el paciente muestra síntomas o es posible una exposición reciente, debe reprogramar su cita para servicios no urgentes o quirúrgicos. Instruir al paciente para que acuda a consulta solo. Debido a las medidas de vigilancia adicionales, dejar un margen de tiempo a la llegada. Vigilar también a cualquier acompañante que visite la consulta. Realizar el proceso de registro de la forma más virtual. Enviar por correo electrónico formas seguras al paciente para solicitarle que llene todo el papeleo requerido antes de la visita. Adoptar la distancia social cuando salude a los pacientes con asentimientos, sonrisas o movimientos de la mano. Debe evitarse dar la mano o abrazar. Determinar si algún procedimiento a realizar en el día requiere EPP adicional, como procedimientos ablativos de láser o dermoabrasión. La mayor parte de los procedimientos dermatológicos no emite aerosol o gotas.

III. Después de la consulta

1. Continuar las consultas a distancia (si aún hay restricciones) durante el tiempo más tranquilo de la práctica. 


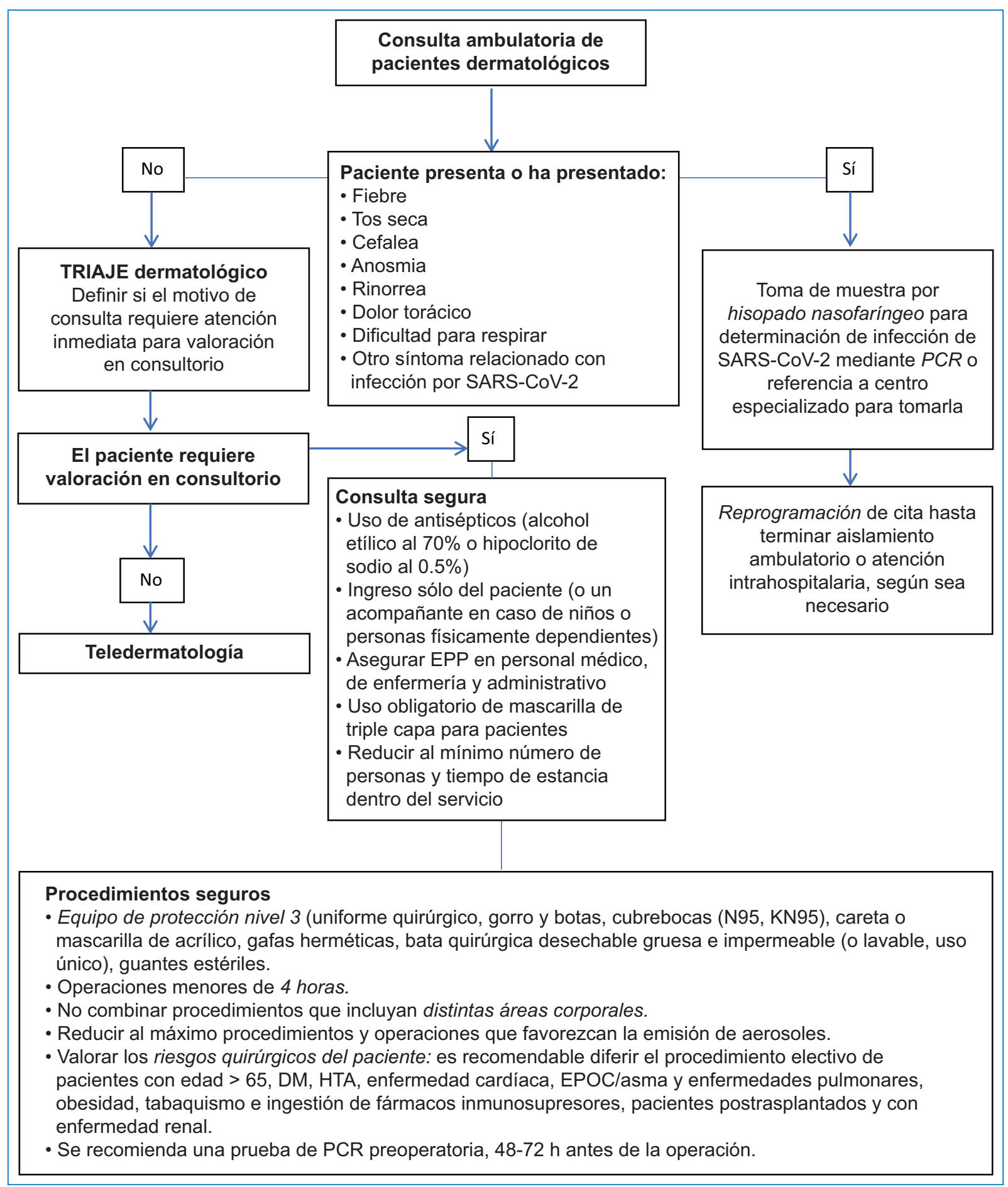

Figura 1. Algoritmo propuesto para la práctica de consulta segura en dermatología.

2. Reducir al mínimo el seguimiento en persona con uso de suturas absorbibles u ocultas para los procedimientos quirúrgicos.

3. Realizar las consultas de seguimiento a distancia cuando sea práctico. Ante la ausencia de citas en línea, considerar contratar una plataforma virtual para que los pacientes puedan programar sus citas más fácilmente y el personal no se sobrecargue con llamadas para la demanda de confinamiento acumulada. 
IV. Procedimientos en consultorio.

En procedimientos de consultorio, como inyectables, exfoliaciones, microagujas, láseres y servicios de limpiezas faciales, se recomienda al personal utilizar equipo de protección de nivel 3 , es decir, uniforme quirúrgico, gorro y botas, cubrebocas (N95, KN95), careta o mascarilla de acrílico, gafas herméticas, bata quirúrgica desechable gruesa e impermeable (o lavable que debe cambiarse con cada paciente, uso único), guantes estériles ${ }^{1-5}$.

En cuanto a los procedimientos quirúrgicos dermatológicos y estéticos, el objetivo es efectuar procedimientos electivos en pacientes sanos y reducir el tiempo de exposición del personal durante la intervención, para lo cual se recomienda ${ }^{2-5}$ :

1. Operaciones menores de 4 horas.

2. Reducir al máximo los procedimientos e intervenciones que produzcan emisiones de aerosoles.

Nota: el aerosol es un coloide de partículas sólidas o líquidas suspendidas en el aire que pueden medir desde $0.002 \mu \mathrm{m}$ hasta más de $100 \mu \mathrm{m}$. La transmisión viral se facilita cuando se realizan procedimientos o se administran tratamientos que liberan aerosoles, entre ellos la intubación endotraqueal, broncoscopia, aspiración abierta, administración de un fármaco por nebulización, ventilación manual antes de la intubación, giro del paciente al decúbito prono, desconexión de un ventilador, ventilación no invasiva con presión positiva, traqueostomía y reanimación cardiopulmonar. Es importante señalar que en dermatología aún no hay evidencia de la viabilidad del SARS CoV-2/COVID-19 después de practicar procedimientos como los de electrocirugía, criocirugía y láser.

3. Valorar los riesgos quirúrgicos del paciente. Es recomendable diferir la operación electiva de pacientes > 65 años, diabetes mellitus, hipertensión arterial, enfermedad cardíaca, EPOC (enfermedad pulmonar obstructiva crónica)/asma y enfermedades pulmonares, apnea obstructiva del sueño, obesidad e ingestión de fármacos inmunosupresores, pacientes trasplantados, con enfermedad renal (glomerulonefritis/insuficiencia renal, etc.).

4. Es recomendable una prueba de PCR (reacción en cadena de la polimerasa) preoperatoria para determinar la infección aguda de pacientes quirúrgicos electivos antes de la operación.
5. Los procedimientos de drenaje que mejoran las condiciones del paciente (p. ej., incisión y drenaje de abscesos de hidradenitis supurativa) pueden no ser diferibles y realizarse inmediatamente con los cuidados mencionados antes.

6. En oncología dermatológica, y debido al riesgo de avance de la enfermedad, en la medida de lo posible se realizará priorización de todos los tumores, lo cual es fundamental para definir una conducta quirúrgica inmediata o diferida. Las herramientas que deben considerarse son el tipo de tumor y el subtipo histológico. Es importante resaltar los criterios de alto riego de infiltración y recidiva tumoral y el riego de metástasis. En el caso de lesiones melanocíticas que sugieran sospecha de nevo displásico o melanoma in situ, se recomienda la biopsia excisional. Estas recomendaciones se resumen de forma general en la figura 1.

\section{Agradecimientos}

Los autores agradecen a la directiva y miembros del Colegio Ibero-Latinoamericano de Dermatología y la Sociedad Mexicana de Dermatología, A.C.

\section{Conflicto de intereses}

Los autores declaran que no existe conflicto de intereses.

\section{Financiamiento}

Apoyo irrestricto e incondicional de Laboratorios Galderma.

\section{Responsabilidades éticas}

Protección de personas y animales. Los autores declaran que para esta investigación no se han realizado experimentos en seres humanos ni en animales.

Confidencialidad de los datos. Los autores declaran que en este artículo no aparecen datos de pacientes.

Derecho a la privacidad y consentimiento informado. Los autores declaran que en este artículo no aparecen datos de pacientes. 
Med Cutan Iber Lat Am. 2020;48(2)

\section{Bibliografía}

1. American Academy of Dermatology [Internet]. Coronavirus Resource Center. 2020. [citado 3 octubre 2020]. Disponible en: https://www.aad. org/member/practice/coronavirus/reopening-your-dermatology-practice

2. Al-Niaimi F, Ali FR. COVID-19 and dermatologic surgery: hazards of surgical plume. Dermatol Ther. 2020;e13593.

3. British Association of Dermatologists and British Society for Dermatological Surgery. Clinical Guidance for the Management of Skin Cancer Patients During the Coronavirus Pandemic. 2020. [citado 3 octubre 2020]. Disponible en: http://www.bad.org.uk/shared/getfile.ashx?itemtype=document\&id=6658
4. Melanoma Institute Australia. Important notice regarding the management of clinically suspected primary melanoma during the COVID-19 crisis. 2020. [citado 3 octubre 2020]. Disponible en: https:// mcusercontent.com/88a3bd528a963791abea7c880/files/b853272a 052d-4eeb-b62a-ea0bd2cbce60/Melanoma_Management__Covid_ FINAL.pdf

5. British Association of Dermatologists \& British Society for Dermatological Surgery. Clinical guidance for the management of skin cancer patients during the coronavirus pandemic. 2020. [citado 3 octubre 2020]. Disponible en: https://www.bsds.org.uk/uploads/COVID19\%20-\%20Guidance\%20for\%20skin\%20cancer\%20surgery\%2030.03.20\%20FINAL.pdf 